\title{
Relação da síndrome metabólica com o câncer de mama
}

\author{
Daniel Buttros ${ }^{1}$, Gilberto Uemura², Eliana Petri Nahás³
}

A síndrome metabólica (SM) sabidamente associa-se com o aumento no risco de desenvolvimento da doença cardiovascular (DCV) e diabetes tipo II $^{1}$. É definida como um conjunto de fatores de riscos metabólicos que incluem obesidade abdominal, dislipidemia, hipertensão arterial e disglicemia ${ }^{2}$. Acomete aproximadamente $30 \%$ da população de mulheres acima dos 50 anos, com aumento de 3 vezes o risco de morbimortalidade por $\mathrm{DCV}^{3-5}$. Observa-se aumento progressivo da prevalência da SM com o envelhecimento.

Em 2001, US National Cholesterol Education Program: Adult Treatment Panel III (NCEP-ATP III) propôs o diagnóstico da SM considerando a presença de três dos cinco critérios diagnósticos: obesidade abdominal (circunferência da cintura), elevaçấo da pressão arterial, reduçấo de HDL-colesterol, aumento de triglicerídeos e intolerância a glicose $e^{6}$.

Estratégias atuais no diagnóstico e tratamento do câncer de mama têm contribuído para o aumento na sobrevida de pacientes oncológicas ${ }^{7,8}$. Com isso, comorbidades incluindo as DCV emergem como causas competitivas de morte ao longo da vida dessas mulheres. Nichols et al. analisaram dados de uma coorte de 3.993 mulheres norte-americanas com idade entre 25 e 79 anos com diagnóstico de câncer de mama não metastático. Durante um período médio de seguimento de 6,3 anos, os autores identificaram 121 mortes pelo câncer e 95 mortes por DCV. Entre as mulheres que ganharam peso pós-diagnóstico, cada $5 \mathrm{~kg}$ de ganho esteve associado a $13 \%$ de aumento na mortalidade por câncer e a $19 \%$ por DCV 9 .

Estudo desenvolvido na Faculdade de Medicina de Botucatu, São Paulo, demonstrou que pacientes na pós-menopausa tratadas de câncer de mama apresentaram aumento do risco de desenvolver SM, com odds ratio (OR) de 1,66 e intervalo de confiança de 95\% (IC95\%) 1,04-2,68, disglicemia (OR=1,05; IC95\% 1,09-3,03) e hipertensão arterial (OR=1,71; IC95\% 1,02-2,89) quando comparadas às mulheres de mesma faixa etária sem a doença. A SM esteve presente em $50 \%$ das pacientes tratadas de câncer de mama e em $37,5 \%$ no grupo controle ${ }^{10}$. Esses resultados estão em concordância com outros estudos ${ }^{11,12}$.

Mulheres com SM apresentam maiores taxas de estadios II, III e IV no momento do diagnóstico do câncer de mama quando comparadas às pacientes sem síndrome, sendo as taxas de 78 e 55\%, respectivamente. A hiperinsulinemia, a hiperglicemia e a obesidade central apresentam relaçấo direta com o comprometimento axilar, alto grau histológico e estadiamento tardio no diagnóstico do câncer de mama ${ }^{13}$.

Em grande estudo populacional, Rosato et al. compararam 3.869 mulheres na pós-menopausa com câncer de mama com 4.082 mulheres na mesma faixa etária sem câncer. Os fatores de risco significativos para câncer de mama foram: diabete mellitus, hipertensão arterial, hiperlipidemia e obesidade abdominal. Os autores concluíram que a SM é fator de risco independente para câncer de mama, com risco relativo (RR) 1,75, e que o risco aumenta com a idade $(\mathrm{RR}=3,04)^{14}$.

A literatura recente associa a SM ao câncer de mama em diferentes aspectos:

1. SM aumenta o risco de câncer de mama na pós-menopausa ${ }^{14}$;

2. mulheres com câncer de mama na pós-menopausa têm maior risco de desenvolver $S M^{10}$;

3. mulheres com SM e câncer de mama têm pior prognóstico oncológico ${ }^{13}$;

4. mulheres com SM e câncer de mama têm alto risco de morte por $\mathrm{DCV}^{9}$.

"Mestre em Ginecologia, Obstetrícia e Mastologia pela Faculdade de Medicina de Botucatu da Universidade Estadual Paulista "Júlio de Mesquita Filho" (UNESP) - Botucatu (SP), Brasil.

${ }^{2}$ Professor Doutor da Disciplina de Mastologia da Faculdade de Medicina de Botucatu da UNESP - Botucatu (SP), Brasil.

${ }^{3}$ Professora Livre Docente do Departamento de Ginecologia e Obstetrícia da Faculdade de Medicina de Botucatu da UNESP - Botucatu (SP), Brasil. Endereço para correspondência: Daniel Buttros - Avenida Trinta e Três - 102 - CEP: 13501-150 - Rio Claro (SP), Brasil -

E-mail: danielbuttros@hotmail.com 
Dados da SM em relação ao câncer de mama na pós-menopausa são bem demonstrados; porém, na pré-menopausa, ainda são escassos. Estudo de destaque apresentado no congresso da American Society of Clinical Oncology/2014 (ASCO) concluiu que a obesidade (IMC $\geq 30 \mathrm{~kg} / \mathrm{m}^{2}$ ) é fator de risco independente para morte em mulheres com câncer de mama inicial, receptor de estrogênio positivo, na pré e perimenopausa ${ }^{15}$.

Após o diagnóstico da SM, a conduta direciona-se a reduzir fatores causais, como a obesidade e inatividade física, e ao tratamento associado dos fatores lipídicos (dislipidemias) e não lipídicos (hipertensão arterial) ${ }^{6}$. O tratamento deve ser intensivo com mudança de hábitos, incluindo reduçáo na ingestão de gordura saturada e colesterol, aumento da atividade física e controle do peso corporal ${ }^{16-18}$.

Devido aos conceitos recentes sobre a relação entre a hiperinsulinemia, a SM e o câncer de mama, a metformina tem sido intensamente estudada na ciência oncológica ${ }^{19-21}$. Já associada com o aumento da taxa de resposta patológica completa em pacientes com câncer de mama que fizeram quimioterapia neoadjuvante em vigência da droga, a metformina surge como possível droga na quimioprevenção primária do câncer de mama ${ }^{22}$.

\section{Referências}

1. Solymoss BC, Bourassa MG, Marcil M, Levesque S, Varga S, Campeau L. Long-term rates of cardiovascular events in patients with metabolic syndrome according to severity of coronary-angiographic alterations. Coron Artery Dis. 2009;20(1):1-8.

2. Schneider JG, Tompkins C, Blumenthal RS, Mora S. The metabolic syndrome in women. Cardiol Rev. 2006;14(6):286-91.

3. Meigs JB. Epidemiology of the metabolic syndrome. Am J Manag Care. 2002;8(Suppl 11):283-92.

4. Ford ES, Giles WH, Mokad AH. Increasing prevalence of the metabolic syndrome among US adults. Diabetes Care. 2004;27;2444-9.

5. Nahas EAP, Padoani NP, Nahas-Neto J, Orsatti FL, Tardivo AP, Dias R. Metabolic syndrome and its associated risk factors in Brazilian postmenopausal women. Climacteric. 2009;12(5):431-8.

6. NCEP Expert Panel on the detection, evaluation, and treatment of high blood pressure in adults. Executive summary of the Third Report of the National Cholesterol Education Program (NCEP). Adult Treatment Panel III (ATP III). JAMA. 2001;285:2444-9.

7. Ganz PA. Survivorship: Adult Cancer Survivors. Prim Care Clin Office Pract. 2009;36:721-41.

8. Geiger S, Lange V, Suhl P, Heinemann V, Stemmler HJ. Anticancer therapy induced cardiotoxicity: review of the literature. Anti-Cancer Drugs. 2010;21:578-90.

9. Nichols HB, Trentham-Dietz A, Egan KM, , Titus-Ernstoff L, Holmes MD, Bersch AJ, et al. Body mass index before and after breast cancer diagnosis: associations with all-cause, breast cancer, and cardiovascular disease mortality. Cancer Epidemiol Biomarkers Prev. 2009;18(5):1403-9.

10. Buttros D, Nahas EA, Vespoli HL, Uemura G, de Almeida Bda R, Nahas-Neto J. Risk of metabolic syndrome in postmenopausal breast cancer survivors. Menopause. 2013;20(4):448-54.

11. Thomson CA, Thompson PA, Wright-Bea J, Nardi E, Frey GR, Stopeck A. Metabolic syndrome and elevated C-reactive protein in breast cancer survivors on adjuvant hormone therapy. J Women Health. 2009;18:2041-7.

12. Porto LA, Lora KJ, Soares JC, Costa LO. Metabolic syndrome is an independent risk factor for breast cancer. Arch Gynecol Obstet. 2011; 284(5):1271-6.

13. Healy LA, Ryan AM, Carroll P, Ennis D, Crowley V, Boyle T, et al. Metabolic syndrome, central obesity and insulin resistance are associated with adverse pathological features in postmenopausal breast cancer. Clin Oncol. 2010;22:281-8.

14. Rosato V, Bosetti C, Talamini R, Levi F, Montella M, Giacosa A, et al. Metabolic syndrome and the risk of breast cancer in postmenopausal women. Ann Oncol. 2011;22(12):2687-92.

15. Pan H, Gray RG; Early Breast Cancer Trialists' Collaborative Group. Effect of obesity in premenopausal ER+ early breast cancer: EBCTCG data on 80,000 patients in 70 trials. Chicago: ASCO Annual Meeting; 2014.

16. Carr MC. The emergence of the metabolic syndrome with menopause. J Clin Endocrinol Metab. 2003;88:2404-11. 
17. Grundy SM, Hansen B, Smith SC, et al. Clinical management of metabolic syndrome: report of the National Heart, Lung and Blood Institute/American Heart Association Conference on Scientific Issues Related to Definition. Circulation. 2005;109:551-6.

18. Sutherland JP, McKinley B, Eckel RH. The metabolic syndrome and inflammation. Metab Syndr Relat Disord. 2004;2(2):82-104

19. Yang YX, Hennessy S, Lewis JD. Insulin therapy and colorectal cancer risk among type 2 diabetes mellitus patients. Gastroenterol. 2004;127:1044-50.

20. Lipscombe LL, Goodwin PJ, Zinman B, McLaughlin JR, Hux JE. Increased prevalence of prior breast cancer in women with newly diagnosed diabetes. Breast Cancer Res Treat. 2006;98(3):303-9.

21. Bodmer M, Meier C, Krahenbuhl S, Jick SS, Meier CR. Long-term metformin use is associated with decreased risk of breast cancer. Diabetes Care. 2010;33(6):1304-8.

22. Jiralerspong S, Palla SL, Giordano SH, Meric-Bernstam F, Liedtke C, Barnett CM, et al. Metformin and pathologic complete responses to neoadjuvant chemotherapy in diabetic patients with breast cancer. J Clin Oncol. 2009;27(20):3297-302. 